\title{
Value of combined detection of PCA, ANCA, ASCA, AGA and ANA in early diagnosis of Gastrointestinal Diseases
}

\author{
Xin $\mathrm{Liu}^{1}$, Deli Guo², Xiaoyan $\mathrm{Li}^{3}$, \\ Yaping Guo ${ }^{4}$, Wenjie Song ${ }^{5}$
}

\begin{abstract}
Objectives: To investigate the positive detection rate and clinical application value of anti-parietal cell antibody (PCA), anti-neutrophil cytoplasmic antibody (ANCA), anti-Saccharomyces cerevisiae antibody (ASCA), anti-gliadin antibody (AGA) and anti-nuclear antibody (ANA) in patients visiting the Department of Gastroenterology.

Methods: From January 2015 to June 2018, 1,083 patients receiving detection of PCA and other autoantibodies were selected from the Department of Gastroenterology of Baoding First Central Hospital. The positive detection rate of autoantibodies was statistically analyzed. The enumeration data were expressed as rate or constituent ratio, and the rates were compared between groups using the $\mathrm{x}^{2}$ test.

Results: Among the 1,083 patients, the positive detection rate of ANA, ASCA, AGA, PCA and ANCA was $33.52 \%, 16.71 \%, 15.51 \%, 13.66 \%$ and $7.66 \%$, respectively. The total positive detection rate was $62.8 \%$ (n $=680$ ).

Conclusion: The population with abdominal distension, chronic abdominal pain, diarrhea and other digestive system symptoms should be timely treated with combined detection of PCA, ANCA, ASCA, AGA and ANA, which is of important clinical application value for early diagnosis of gastrointestinal diseases and prevention of missed diagnosis and misdiagnosis.
\end{abstract}

KEYWORDS: Anti-parietal cell antibody, Anti-neutrophil cytoplasmic antibody, Anti-Saccharomyces cerevisiae antibody, Anti-gliadin antibody; Anti-nuclear antibody.

doi: https://doi.org/10.12669/pjms.38.1.4682

How to cite this:

Liu X, Guo D, Li X, Guo Y, Song W. Value of combined detection of PCA, ANCA, ASCA, AGA and ANA in early diagnosis of Gastrointestinal Diseases. Pak J Med Sci. 2022;38(1):227-231. doi: https://doi.org/10.12669/pjms.38.1.4682

This is an Open Access article distributed under the terms of the Creative Commons Attribution License (http://creativecommons.org/licenses/by/3.0), which permits unrestricted use, distribution, and reproduction in any medium, provided the original work is properly cited.

1. Xin Liu,

Department of Clinical Laboratory,

2. Deli Guo,

Department of Orthopedics,

3. Xiaoyan $\mathrm{Li}$

Department of Clinical Laboratory,

4. Yaping Guo,

Department of Clinical Laboratory,

5. Wenjie Song,

Department of Clinical Laboratory,

1-5: Baoding NO.1 Central Hospital, Baoding, 071000, Hebei, China.

Note: Xin Liu and Deli Guo are both considered as co-first authors.

Correspondence:

Yaping Guo,

Department of Clinical Laboratory,

Baoding No.1 Central Hospital,

Baoding, 071000, Hebei, China.

E-mail: guoyapingjyk01@163.com

* Received for Publication:

May 6, 2021

* Revision Received:

* Revision Accepted: *

August 20, 2021

August 30, 2021

\section{INTRODUCTION}

Abdominal distension, chronic abdominal pain and diarrhea are common symptoms of patients visiting the Department of Gastroenterology, which are not specific and can be caused by multiple diseases. The factors causing gastrointestinal diseases are numerous, and the etiology is complex. The common ones include infection, physicochemical factors, metabolic and absorption disorders, autoimmunity, etc., among which autoimmune factors play an important role. ${ }^{1}$ Autoantibodies refer to the antibodies against the tissues, organs, cells and cell components of the body, which are immunoglobulins produced by the immune response to the body's own components due to immune dysfunction. With 
the development of detection technologies and the development and application of various autoantibodies, the incidences of autoimmune gastrointestinal diseases such as inflammatory bowel disease (IBD), type $\mathrm{A}$ atrophic gastritis (AIG) and celiac disease have been increasing in recent years. ${ }^{2-4}$ Because of atypical early symptoms and similar clinical manifestations, their diagnosis mostly depends on endoscopy and pathology, and pathological development is a slow and gradual process, always resulting in diagnosis many years after the symptoms appear and very severe outcomes in many cases. Therefore, it is of great significance for the precise diagnosis and treatment of diseases to accurately determine the types of diseases in the early stage by serological autoantibody detection. In this paper, the combined detection results of PCA, ANCA, ASCA, AGA and ANA in the Department of Gastroenterology of our hospital were analyzed and discussed.

\section{METHODS}

From January 2015 to June 2018, 1,083 patients receiving detection of PCA, ANCA, ASCA, AGA and ANA were selected from the Outpatient and Inpatient Department of Gastroenterology of Baoding First Central Hospital. Exclusion criteria included: patients with liver diseases; patients with primary hematological diseases; and patients with mental illness.

Ethical Approval: The study was approved by the Institutional Ethics Committee of Baoding No.1 Central Hospital on March 01, 2021(No.:[2021011]), and written informed consent was obtained from all participants.

Equipment and reagents: $\mathrm{BX} 43$ fluorescence microscope was from Olympus (Japan), and MULTSKAN FC microplate reader was from ThermoFisher Instrument Co., Ltd. (Shanghai). Detection reagents for ANA and ANCA were from EUROIMMUN Medical Diagnostics Co., Ltd., and detection reagents for ACSA, AGA and PCA were from Guangzhou Kangrun Biotechnology Co., Ltd.
Table-I: Positive detection rate of ANA, ASCA, AGA, PCA and ANCA.

\begin{tabular}{llc}
\hline Item & $N$ & Positive detection rate, $\%$ \\
\hline ANA & 363 & 33.52 \\
ASCA & 181 & 16.71 \\
AGA & 168 & 15.51 \\
PCA & 148 & 13.66 \\
ANCA & 83 & 7.66 \\
\hline
\end{tabular}

ANA and ANCA were detected using indirect immunofluorescence, and PCA, ASCA and AGA were detected by Western blot. The operation was strictly in accordance with the instructions. For the subjects, present situation investigation and retrospective analysis were mainly used.

Statistical Analysis: Statistical analysis was carried out using SPSS19.0. The enumeration data were expressed as rate or constituent ratio, and the rates were compared between groups using the $\mathrm{x}^{2}$ test. $P<0.05$ was considered as statistically significant.

\section{RESULTS}

Among the 1,083 patients, 680 patients showed five positive antibodies, with the total positive detection rate of $62.8 \%$. The positive detection rate of ANA was the highest $(33.52 \%)$. The positive detection rate of each antibody is shown in Table-I.

A total of 480 patients presented positive PCA, ASCA, AGA and ANCA, with the positive detection rate of $44.32 \%$. Among them, ANA was positive in 163 patients, accounting for $33.96 \%$. ASCA, AGA and ANCA were negative in 603 patients, among which ANA was positive in 200 patients, accounting for $33.167 \%$. No statistically significant differences were found in the positive detection rate of ANA between the autoantibodypositive group and the autoantibody-negative group, as seen in Table-II.

Table-II: Combined detection results of ANA between autoantibody-positive group and autoantibody-negative group.

\begin{tabular}{ccccc}
\hline & Positive number of ANA & Total number & $\chi^{2}$ & $P$ \\
\hline Autoantibody-positive group & 163 & 480 & 0.08 & $>0.05$ \\
Autoantibody-negative group & 200 & 603 & & \\
\hline
\end{tabular}




\section{DISCUSSION}

The positive detection rate of ANA and other autoantibodies was $62.8 \%$, suggesting that autoimmune factors account for a high proportion in gastrointestinal diseases. Previously, it was reported that the prevalence rate of gastrointestinal autoimmune diseases was very low, and some were even rare diseases. ${ }^{5}$ In this study, it was found that 680 of 1,083 patients in the Department of Gastroenterology had positive ANA and other autoantibodies. The causes may be analyzed as follows:

1. Autoimmunity plays an important role in the occurrence and development of diseases;

2. Because the early symptoms of gastrointestinal diseases are not typical and pathology is a process of slow progression, many gastrointestinal AID are not timely diagnosed, resulting in missed diagnosis and misdiagnosis;

3. PCA and other autoantibodies also have a certain positive rate in non-AID diseases. Therefore, PCA and other autoantibodies have important application value in the diagnosis and differential diagnosis of gastrointestinal diseases.

In this study, it was found that the positive detection rate of ANA was $33.52 \%$ in the patients visiting the Department of Gastroenterology, which was lower than that in our previous study based on a large sample size. ${ }^{6}$ ANA is the general term of autoantibodies targeting various components of eukaryotic cells as target antigens. ANA acts on mitotic cell cycle, resulting in production disorders of DNA and proteins and blocked metabolism, so it has strong damage to the tissues with exuberant metabolism. ANA detection is a classic screening test for AID. There is high titer of ANA in the serum of AID patients. In recent years, a study ${ }^{8}$ has found that ANA exists in the serum of patients before clinical symptoms appear, which is predictive for diseases. Gastrointestinal vasculitis caused by systemic lupus erythematosus is the most common cause of gastrointestinal involvement. ${ }^{9}$ The pathogenesis includes the deposition of immune complexes and complements of small vessels in the mesentery and intestinal wall, accompanied by inflammatory cell infiltration, and thrombosis in small vessels, which results in thickening and occlusion of the wall of small mesenteric artery, causing intestinal mucosal edema, intestinal ischemia, ulcers, bleeding intestinal obstruction, perforation, etc. ${ }^{10}$
One third of the patients with positive ANCA, PCA, ASCA and AGA were accompanied with positive ANA. Whether these patients have the possibility of overlap syndrome or specific markers of certain AID involving the gastrointestinal tract remains to be further explored.

The positive detection rate of ASCA was $16.71 \%$. The main target antigen of ASCA is phosphopeptide mannan on the cell wall of Saccharomyces cerevisiae (homologous with cell wall component of intestinal flora). ASCA is involved in the pathogenesis of Crohn's disease (CD), which is a highly specific serum marker for CD patients. ${ }^{11}$ The target antigens of ANCA are various components in the cytoplasm of neutrophils and monocytes. ${ }^{12}$ Protease 3 and myeloperoxidase are considered to be the target antigens of vasculitis. In IBD, the target antigen corresponding to pANCA is histone 1 , and pANCA is considered to be an autoantibody induced by cross reaction with intestinal bacterial antigens. ${ }^{13}$ pANCA can be detected in $60-70 \%$ patients with ulcerative colitis (UC). IBD is still a rare disease in China, but its incidence has been increasing rapidly in recent 20 years. It has been shown ${ }^{14}$ that the incidence of UC (1.64/10 million) in China is higher than that of CD $(0.13 / 10$ million), while the missed diagnosis rate of $C D$ is $60.9 \%$, which is nearly 1 time higher than that of UC (32.1\%). However, our study revealed that the positive detection rate of ASCA was $16.77 \%$, which was higher than that of ANCA (7.66\%), confirming that many patients with CD may not be diagnosed, and the missed diagnosis is not optimistic.

The target antigens of AGA is a group of proteins rich in proline and glutamine, which are the main toxic components of gluten. These proteins can not be digested in the gastrointestinal tract. Through connexin, the cytoskeleton is reconstructed and the tight junction is destroyed, leading to increased permeability of the small intestinal mucosa. The toxic effect of gliadin also reduces the fibroactin of small intestinal epithelial cells, inhibits the growth of epithelial cells, and induce apoptosis. ${ }^{15}$ AGA is a serological marker for celiac disease. The prevalence rate of celiac disease is much higher than expected, and only $10-15 \%$ of the patients are diagnosed. ${ }^{16}$ This investigation found that its positive detection rate in patients in the Department of Gastroenterology was $15.51 \%$, which was far higher than its prevalence rate. The prevalence rate of celiac disease is higher in 
the West. ${ }^{17}$ With the development of society, the change of dietary structure and the improvement of laboratory detection technology, the prevalence rate of celiac disease in China is increasing.

The target antigens of PCA are $\mathrm{H}^{+} / \mathrm{K}^{+}$-ATPase and gastrin receptor on the surface of gastric parietal cells. After PCA formation, the mucosa of the gastric body and fundus where gastric parietal cells are located is damaged and atrophied, the glands are decreased, and then the function is reduced, leading to dyspepsia symptoms such as abdominal distension, and forming chronic atrophic gastritis after a long term. Chronic atrophic gastritis is a common clinical disease in China, without gold standard for its diagnosis. ${ }^{18}$ PCA-positive is the characteristic of type A atrophic gastritis, which mainly causes atrophy of the gastric body. Helicobacter pylori infection can lead to chronic non-atrophic gastritis and atrophic gastritis, mostly presenting multifocal gastric mucosal atrophy, which is called type B atrophic gastritis. The rate of Helicobacter pylori infection is high in China. In the past, it was believed that atrophic gastritis in China was mainly type B atrophic gastritis, and type $\mathrm{A}$ atrophic gastritis was rare. ${ }^{19}$ Our study found that the positive detection rate of PCA was $13.66 \%$, which was much higher than the prevalence rate. It has been reported that Helicobacter pylori can activate selfreactive $T$ lymphocytes and trigger autoimmune mechanism through the molecular simulation mechanism at the level of $\mathrm{T}$ lymphocytes, thus leading to type A atrophic gastritis. ${ }^{20}$ Therefore, the prevalence rate of type A atrophic gastritis is likely to be underestimated, mainly caused by the high incidence of Helicobacter pylori infection in China, the lack of PCA detection and the gradual process of pathological changes. Consequently, PCA detection can be used as a routine screening of gastritis, which is of great significance for clarifying the etiology and early prevention and treatment.

IBD, celiac disease, atrophic gastritis and other autoimmune gastrointestinal diseases have been increasing in recent years in China, but their incidences are still low. Without gold standard, they are mainly comprehensively diagnosed based on clinical manifestations, laboratory indexes, endoscopic and pathological results. The early clinical manifestations of these autoimmune gastrointestinal diseases are similar, and the pathological progression is a gradual process, which leads to the high rate of missed diagnosis and misdiagnosis. However, in our study, it was revealed that the positive detection rates of specific serological markers for these diseases were much higher than their prevalence rates, proving that these markers already exist in the serum of patients before diagnosis, and can be used as a good detection method, which has important value for the early diagnosis of the diseases. Therefore, the detection rate of these diseases can be improved by expanding the detection range of autoantibodies and clinical combined detection.

Limitations of this study: Our sample size is not large enough. In future studies, countermeasures are being taken to further accumulate patient information, increase the sample size, extend the follow-up time, and carry out more stringent experimental design, so as to make up for the above shortcomings and be more conducive to guiding clinical practice.

\section{CONCLUSION}

The population with abdominal distension, chronic abdominal pain, diarrhea and other digestive system symptoms should be timely treated with combined detection of PCA, ANCA, ASCA, AGA and ANA, which is of important clinical application value for early diagnosis of gastrointestinal diseases and prevention of missed diagnosis and misdiagnosis.

\section{Conflicts of interest: None.}

Source of funding: The study was sponsored by Baoding Key Laboratory of research on the pathogenicity and predictive value of autoantibodies (No.SZ2020002).

\section{REFERENCES}

1. Chen LL, Chen SS, NI XQ, Zhang JJ, Tian QX. Effect of rocuronium on postoperative infections in patients undergoing gastrointestinal surgery. Chinese J Nosocomiol. 2017;27(22):5156-5159.

2. Fang JY, Du YQ, Liu WZ, Ren JL, Li YQ, Chen XY, et al. Chinese consensus on chronic gastritis (2017, Shanghai) [published correction appears in J Dig Dis. 2020;21(2):120. J Dig Dis. 2018;19(4):182-203. doi: 10.1111/1751-2980.12593

3. Kotze PG, Underwood FE, Damião AOMC, Ferraz JGP, Saad-Hossne R, Toro M, et al. Progression of Inflammatory Bowel Diseases Throughout Latin America and the Caribbean: A Systematic Review. Clin Gastroenterol Hepatol. 2020;18(2):304-312. doi: 10.1016/j.cgh.2019.06.030

4. Penny HA, Raju SA, Sanders DS. Progress in the serologybased diagnosis and management of adult celiac disease. Expert Rev Gastroenterol Hepatol. 2020;14(3):147-154. doi: 10.1080/17474124.2020.1725472. 
5. Kou GJ, Guo J, Zuo XL, Li CQ, Liu C, Ji R, et al. Prevalence of celiac disease in adult Chinese patients with diarrheapredominant irritable bowel syndrome: A prospective, controlled, cohort study. J Dig Dis. 2018;19(3):136-143. doi: 10.1111/1751-2980.12587

6. Guo YP, Wang CG, Liu X. Antinuclear antibody in health check-up and clinical visit adults. Chinese J Health Management. 2013;7(4):281-284.

7. Committee of Autoantibody Detection of Rheumatology and Immunology Physicians Association of Chinese Medical Doctor Association. Expert consensus on clinical application of antinuclear antibody detection. Chinese of Lab Med. 2018;41(4):275-280.

8. Arbuckle MR, McClain MT, Rubertone MV, Scofield RH, Dennis GJ, James JA, et al. Development of autoantibodies before the clinical onset of systemic lupus erythematosus. N Engl J Med. 2003;349(16):1526-1533. doi: 10.1056/NEJMoa021933

9. Li Z, Xu D, Wang Z, Wang Y, Zhang S, Li M, et al. Gastrointestinal system involvement in systemic lupus erythematosus. Lupus. 2017;26(11):1127-1138. doi: $10.1177 / 0961203317707825$

10. Trapani S, Rubino C, Simonini G, Indolfi G. Gastrointestinal and hepatic involvement in paediatric systemic lupus erythematosus. Clin Exp Rheumatol. 2021;39(4):899-906.

11. Tian Y, Wang HH. The application value of serological detection of ASCA and ANCA serum level in the diagnosis and treatment of inflammatory bowel disease. Chinese J Lab Med. 2018;41(10):708-711.

12. Committee of Autoantibody Detection of Rheumatology and Immunology Physicians Association of Chinese Medical Doctor Association. Expert consensus on clinical application of anti-neutrophil cytoplasmic antibody detection. Chinese J Lab Med. 2018;41(9):644-650.

13. Mitsuyama K, Niwa M, Takedatsu H, Yamasaki H, Kuwaki $\mathrm{K}$, Yoshioka S, et al. Antibody markers in the diagnosis of inflammatory bowel disease. World J Gastroenterol. 2016;22(3):1304-1310. doi: 10.3748/wjg.v22.i3.1304

14. Zhang YJ, Liang $\mathrm{J}, \mathrm{Wu} \mathrm{KC}$. Interpretation of ulcerative colitis in consensus on diagnosis and treatment of inflammatory bowel disease (Beijing, 2018). Chinese J Digestion. 2018;38(5):312-314.
15. Bardella MT, Elli L, Ferretti F. Non Celiac Gluten Sensitivity. Curr Gastroenterol Rep. 2016;18(12):63. doi:10.1007/s11894-016-0536-7.

16. Bai JC, Ciacci C. World Gastroenterology Organisation Global Guidelines: Celiac Disease February 2017 [published correction appears in J Clin Gastroenterol. 2019;53(4):313. J Clin Gastroenterol. 2017;51(9):755-768. doi: 10.1097/MCG.0000000000000919

17. Lohi S, Mustalahti K, Kaukinen $K$, Laurila K, Collin P, Rissanen $\mathrm{H}$, et al. Increasing prevalence of coeliac disease over time. Aliment Pharmacol Ther. 2007;26(9):1217-1225. doi: 10.1111/j.1365-2036.2007.03502.x

18. Li JX, Chen J, Lv B. Consensus on diagnosis and treatment of chronic atrophic gastritis by traditional Chinese medicine combined with Western medicine(2017). Chinese J Integrated Traditional Western Med on Dig. 2018;02:121-131.

19. Sipponen P, Maaroos HI. Chronic gastritis. Scand J Gastroenterol. 2015;50(6):657-667. doi:10.3109/00365521.2 015.1019918

20. Amedei A, Bergman MP, Appelmelk BJ, Azzurri A, Benagiano M, Tamburini C, et al. Molecular mimicry between Helicobacter pylori antigens and $\mathrm{H}+, \mathrm{K}+$--adenosine triphosphatase in human gastric autoimmunity. J Exp Med. 2003;198(8):1147-1156. doi: $10.1084 /$ jem. 20030530

\section{Authors' Contributions:}

XL \& DG: Designed this study and prepared this manuscript, and are responsible and accountable for the accuracy or integrity of the work.

XL and YG: Collected and analyzed clinical data. WS: Significantly revised this manuscript. 